\title{
基于发光共振能量转移的比率型上转换荧光纳米探针检测次硝酸
}

\author{
王培培 ${ }^{a}$ 梁涛 $^{a} \quad$ 左苗苗 ${ }^{b}$ 李贞*, ${ }^{*}$ 刘志洪*, \\ ( ${ }^{a}$ 武汉大学 化学与分子科学学院 武汉 430072) \\ ( ${ }^{b}$ 湖北大学 化学化工学院 武汉 430062)
}

\begin{abstract}
摘要 次硝酸(Nitroxyl, HNO)由一氧化氮(NO)经单电子还原和质子化作用产生, 在生理和病理过程中都有着重要作用. 本工作构建了一种基于发光共振能量转移(Luminescence resonance energy transfer, LRET)的比率型上转换纳米探针用于 检测生物体系中 HNO 含量. 该探针以上转换纳米颗粒(Upconversion nanoparticles, UCNPs)为能量供体, 有机染料 Fl-TP 为能量受体构建. Fl-TP 能特异性识别 HNO 并与之反应生成 Fl-HNO, Fl-HNO 在 400 500 nm 处具有明显的吸收峰, 与 UCNPs 的蓝色发射光谱重叠, 从而发生 LRET 过程. 随着 HNO 浓度增加, Fl-HNO 的荧光强度 $\left(F_{525} \mathrm{~nm}\right)$ 逐渐增强, UCNPs 的荧光强度 $\left(F_{480 \mathrm{~nm}}\right)$ 逐渐下降, 比率信号 $\left(F_{525} \mathrm{~nm} / F_{480 \mathrm{~nm}}\right)$ 逐渐增加, 并与 $\mathrm{HNO}$ 浓度对数呈良好的线性关系, 该探针的线 性检测范围为 $3 \sim 100 \mu \mathrm{mol} \cdot \mathrm{L}^{-1}$, 检出限 $23.4 \mathrm{nmol} \cdot \mathrm{L}^{-1}$. 实验结果表明该比率探针特异性强, 灵敏度高, 并可成功实现 在活细胞和生物组织中 HNO 的检测.
\end{abstract}

关键词 上转换荧光纳米颗粒; 发光共振能量转移; 比率信号; 次硝酸; 苂光成像

\section{A Ratiometric Upconversion Nanoprobe for Detection of HNO Based on Luminescence Resonance Energy Transfer}

\author{
Wang, Peipei $^{a} \quad$ Liang, Tao $^{a} \quad$ Zuo, Miaomiao $^{b} \quad$ Li, Zhen ${ }^{*, b} \quad$ Liu, Zhihong ${ }^{*, a}$ \\ ( ${ }^{a}$ College of Chemistry and Molecular Sciences, Wuhan University, Wuhan 430072) \\ $\left({ }^{b}\right.$ College of Chemistry and Chemical Engineering, Hubei University, Wuhan 430062)
}

\begin{abstract}
Nitroxyl (HNO), produced by nitric oxide (NO) with one-electron reduction and protonation, has recently received substantial interest due to its important roles in various biological functions and pharmacological activities. Research indicates that $\mathrm{HNO}$ also has many potential pharmacological applications for different diseases. Therefore, the development of a reliable method for HNO assay in biosystems is highly desired. Ratiometric fluorescent probes show significant advantages over traditional "turn-on" ones, because simultaneous measurement of two emission signals can provide a built-in correction and thus minimize the inaccurate fluorescence signal readouts. As far as we know, there is no ratiometric fluorescent probe for HNO detection based on upconversion nanoparticles (UCNPs). Herein, a ratiometric nanoprobe for HNO assay was constructed based on the luminescence resonance energy transfer (LRET) principle by using UCNPs with a core-shell structure $\left(\mathrm{NaYbF}_{4}: 30 \% \mathrm{Gd} @ \mathrm{NaYF}_{4}: 2 \% \mathrm{Yb}: 1 \% \mathrm{Tm}\right)$ as the energy donor and an organic dye Fl-TP as the potential energy acceptor. The oleate-coated UCNPs (OA-UCNPs) and Fl-TP were assembled through hydrophobic interaction to construct the upconversion nanoprobe (termed as Fl-TP-UCNPs). Because of the ring-closed form, Fl-TP displayed weak absorption and was non-fluorescent, which blocked the LRET process. After reaction with HNO, the triphenylphosphine moiety left and released Fl-HNO with the fluorescent ring-open form. Fl-HNO showed strong absorption in the range of $400 \sim 500 \mathrm{~nm}$, which completely overlapped with the blue luminescence of UCNPs and triggered the LRET process between UCNPs and Fl-HNO. Thus, the luminescence from UCNPs around $480 \mathrm{~nm}$ decreased and the emission from Fl-HNO around $525 \mathrm{~nm}$ increased with a [HNO]-dependent manner. The ratiometric luminescence intensity $F_{525 \mathrm{~nm}} / F_{480 \mathrm{~nm}}$ showed a good linear relationship $\left(R^{2}=0.9914\right)$ to the logarithm of AS (Angeli's salt, a generally used HNO donor) concentration in the range of $3 \sim$ $100 \mu \mathrm{mol} \cdot \mathrm{L}^{-1}$ and the limit of detection was $23.4 \mathrm{nmol} \cdot \mathrm{L}^{-1}$. The excellent sensitivity, stability, selectivity and low cytotoxicity endow Fl-TP-UCNPs with the superior capability for HNO assay in vitro and in vivo. We found that Fl-TP-UCNPs probe is appropriate for monitoring HNO in living cells as well as imaging HNO in liver tissues. This probe may be a powerful tool for $\mathrm{HNO}$ assay in various physiological processes.
\end{abstract}

Keywords upconversion nanoparticles; luminescence resonance energy transfer; ratiometric signal; nitroxyl; fluorescence bioimaging

* E-mail: zhenli@hubu.edu.cn; zhhliu@whu.edu.cn

Received May 6, 2020; published June 3, 2020.

Supporting information for this article is available free of charge via the Internet at http://sioc-journal.cn.

Project supported by the National Natural Science Foundation of China (Nos. 21625503, 21807028).

项目受国家自然科学基金(Nos. 21625503, 21807028)资助. 


\section{1 引言}

次硝酸 (Nitroxyl, HNO) 由一氧化氮 $(\mathrm{NO})$ 经过单电 子还原和质子化作用产生, 化学性质独特, 在多种生理 和病理过程中发挥着重要作用 ${ }^{[1-4]}$. 研究表明, HNO 可 与生物硫醇反应, 抑制某些含硫醇的酶活性, 如甘油 醛-3-磷酸脱氢酶和乙醛脱氢酶等 ${ }^{[5]}$. HNO 还可刺激心血 管扩张, 因此被作为治疗酗酒和心脏衰竭的潜在药 物 ${ }^{[6-10]}$. 在生理病理状态下, $\mathrm{HNO}$ 含量低, 半衰期短, 极 易发生脱水二聚反应, 检测难度大 ${ }^{[11]}$. 因此, 开发可靠 的用于检测生物系统中 HNO 的分析方法具有极大的应 用价值.

苂光分析法由于具有灵敏度高、非侵入性以及较高 的时空分辨率等优点而被广泛用于生物样品的分析检 测 ${ }^{[12-14]}$. 目前, 检测 HNO 的荧光探针多为有机小分子 探针, 且主要基于将 $\mathrm{Cu}(\mathrm{II})$ 还原为 $\mathrm{Cu}(\mathrm{I})^{[15,16]}$ 或者 2-(二 苯基膦) 苯甲酸与 $\mathrm{HNO}$ 之间发生施陶丁格反应 (Staudinger) ${ }^{[17-20]}$ 的机理构建. 利用施陶丁格反应机理构 建的探针能够有效降低生物还原剂如抗坏血酸、谷胱甘 肽等的影响, 对活性氧类物质 (ROS) 及活性氮类物质 (RNS)均具有较好的选择性 ${ }^{[21-23]}$. 但是这些有机小分子 探针多用可见光激发, 存在生物组织穿透深度低、生物 背景苂光强、易光漂白等问题. 因此, 开发一种新型的、 适于在生物体系特异性检测 HNO 的荧光探针是非常必 要的.

上转换荧光纳米颗粒(Upconversion nanoparticles, UCNPs) 具有近红外光激发, 反斯托克斯发射的特 征 ${ }^{[24,25]}$, 能够有效消除生物样品的自发荧光, 并提高生 物成像的穿透深度. 此外, UCNPs 还具有斯托克斯位移 大、光稳定性好、发射谱带窄等优点, 能有效避免激发 光的干扰且适合长时间成像. 因此, UCNPs 是制备荧光 探针的优良材料, 上转换荧光纳米探针已在生物传感及 成像领域显示出极大的优势 ${ }^{[26-33]}$.

与传统 “turn-on” 类型的探针相比, 比率型探针同 时测量两个发射信号, 可以提供内置校正来降低由于探 针分布不均匀, 样品厚度等问题而产生的测量误差, 提 高在生物体系中检测的准确性 ${ }^{[34-37]}$. 据我们所知, 目前 还没有基于 UCNPs 的比率型 $\mathrm{HNO}$ 探针的报道. 本工作 基于发光共振能量转移(Luminescence resonance energy transfer, LRET) 原理, 以具有独特结构的 UCNPs 为能量 供体, 有机染料 Fl-TP 为潜在能量受体, 成功构建了一 种用于特异性测定 HNO 的比率型纳米探针. 如图 1 所 示, 探针与 HNO 反应后, Fl-TP 转换为 Fl-HNO, 触发 UCNPs 与染料之间的 LRET 过程, 此时 UCNPs 的荧光 被猝灭, 并出现 Fl-HNO 的荧光发射. 比率荧光信号, 即 Fl-HNO 的荧光发射强度 $\left(F_{525 \mathrm{~nm}}\right)$ 与 UCNPs 的上转换 荧光强度 $\left(F_{480 \mathrm{~mm}}\right)$ 的比值, 与 $\mathrm{HNO}$ 浓度的对数呈良好的 线性关系, 可用于 $\mathrm{HNO}$ 的定量检测. 相比于完全依赖 UCNPs 的不同波长发射信号实现比率变化的探针, 这
种基于染料和 UCNPs 发射构建的比率探针与目标物响 应后, UCNPs 荧光信号减弱, 染料荧光信号增强, 比率 信号变化更加明显, 可有效提高探针的检测灵敏度, 使 探针能更真实地反映目标物浓度的变化.

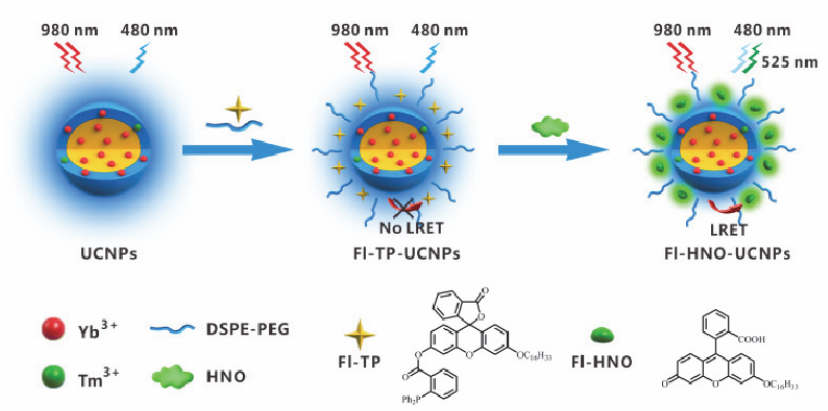

图 1 基于 LRET 原理构建比率型 Fl-TP-UCNPs 探针及对 HNO 响应 的原理图

Figure 1 Schematic illustration of the construction of Fl-TP-UCNPs ratiometric probe and sensing of HNO through LRET principle

\section{2 结果与讨论}

\subsection{FI-TP-UCNPs 的响应机理}

苂光素类染料具有优良的光物理性能, 分子结构易 修饰，很适合作为 UCNPs 的能量受体. 因此，本工作合 成一种苂光素类衍生物 Fl-TP 作为可特异性识别 HNO 的能量受体. 与 $\mathrm{HNO}$ 反应前, Fl-TP 为无吸收的闭环状 态，抑制了 LRET 过程. 与 HNO 发生施陶丁格反应后, Fl-TP 释放三苯基膦单体并转换为开环状态的 Fl-HNO, Fl-HNO 在 $400 \sim 500 \mathrm{~nm}$ 处具有明显吸收(图 2A), 与 UCNPs 的蓝色发射光谱相匹配, LRET 过程得以发生. 由于开环结构的 Fl-HNO 具有强的荧光性能(图 2B), LRET 的发生会同时引起 UCNPs 苂光 $(480 \mathrm{~nm})$ 的减弱及 染料荧光 $(525 \mathrm{~nm})$ 的增强, 从而可根据染料与 UCNPs 荧 光强度的比值进行 HNO 的定量分析.

\subsection{UCNPs 的表征}

本文合成了核壳结构的 UCNPs $\left(\mathrm{NaYbF}_{4}: 30 \% \mathrm{Gd} @\right.$ $\left.\mathrm{NaYF}_{4}: 2 \% \mathrm{Yb}: 1 \% \mathrm{Tm}\right)^{[38-40]}$, 其中 $\mathrm{Gd}^{3+}$ 用于控制晶核生长 速度, 减小纳米颗 粒 粒 径 ${ }^{[41]}$. 与传统结构 $\left(\mathrm{NaYF}_{4}: \mathrm{Yb}, \mathrm{Tm}\right)$ 相比, 这种结构的 UCNPs 具有两个优 势: 首先, 发光离子 $\left(\mathrm{Tm}^{3+}\right)$ 限域在纳米颗粒表面的薄层 内，有效缩短了发光离子与受体间的距离. 由于 LRET 效率与供-受体间距离的六次方成反比 ${ }^{[40,42,43]}$, 距离的 减少可显著提高 LRET 效率; 其次, 核内高浓度掺杂的 敏化剂 $\mathrm{Yb}^{3+}$ 增强了 UCNPs 对近红外光的吸收能力, 能 够显著提高 UCNPs 的亮度 ${ }^{[44]}$. 掺杂在壳层的 $\mathrm{Yb}^{3+}$ 起到 桥联离子的作用, 能提高发光离子的整体泵浦效率, 因 此对壳层中 $\mathrm{Yb}^{3+}$ 浓度进行优化, 确定 $\mathrm{Yb}^{3+}$ 浓度为 $2 \%$ (图 S1). 

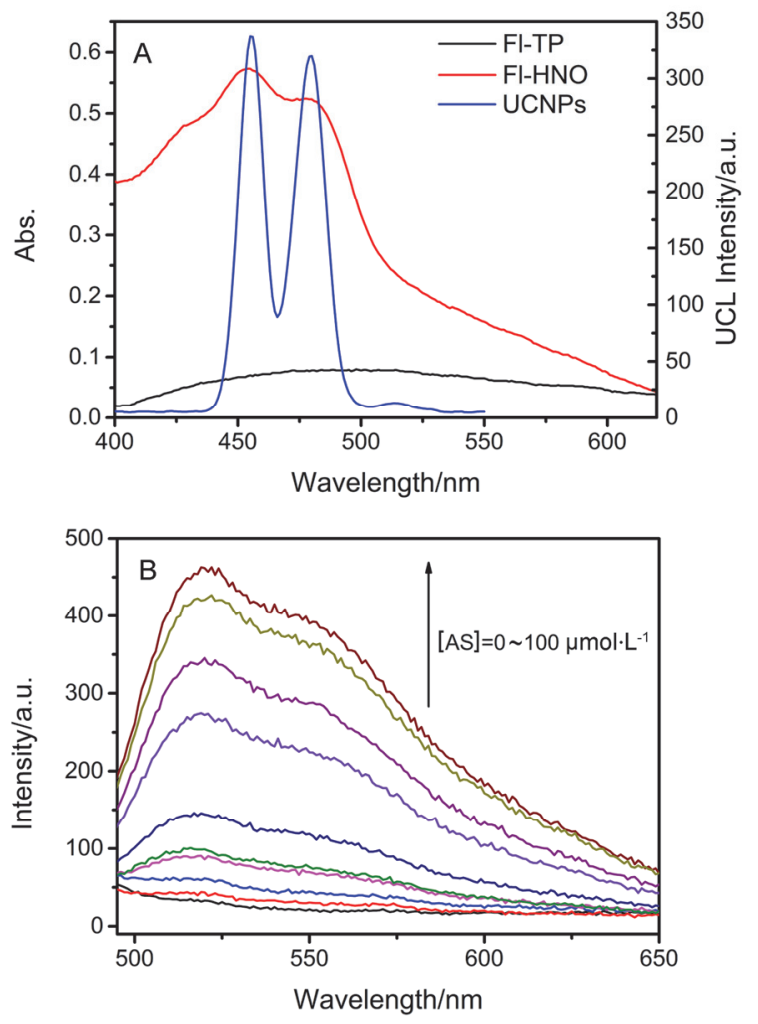

图 2 (A) Fl-TP、Fl-HNO 的吸收光谱和 UCNPs 的发射光谱; (B) Fl-TP 对不同浓度 AS (Angel's salt, HNO 供体)的响应

Figure 2 (A) Absorption spectra of Fl-TP and Fl-HNO as well as the emission spectrum of UCNPs; (B) Emission spectra of Fl-TP responding to different concentrations of AS (Angeli's salt, a generally used HNO donor)

对合成的 UCNPs 进行表征, 透射电子显微镜图像 (Transmission electron microscopy，TEM)表明本文制备 的 UCNPs 具有良好的形貌和均一的尺寸 (图 3A D). 其 中, 核纳米颗粒的粒径为 $(20.0 \pm 1.5) \mathrm{nm}$, 核壳结构 UCNPs 的平均粒径为 $(27.5 \pm 1.7) \mathrm{nm}$, 壳层厚度为 3.8 $\mathrm{nm}$. 所得 UCNPs 的 X 射线粉末衍射(X-ray powder diffraction, XRD)图像与标准六方相 $\mathrm{NaYF}_{4}$ 的谱图(JCPDS No. 16-0034)一致, 表明其为六方相(图 3E).

\subsection{FI-TP-UCNPs 探针的表征}

为实现能量供受体的组装, 两亲性聚合物 DSPE-PEG(分子结构式如图 S2 所示)通过疏水作用包覆 OA-UCNPs, 在 UCNPs 表面形成疏水腔. 随后 Fl-TP 通 过疏水一疏水作用负载于疏水腔内, 完成 Fl-TP-UCNPs 探针的构建 ${ }^{[45]}$. 利用傅里叶变换红外光谱图(Fourier transform infrared spectra, FTIR) 对 Fl-TP-UCNPs 探针进 行表征, 如图 S3 所示, $1769 \mathrm{~cm}^{-1}$ 及 $1640 \mathrm{~cm}^{-1}$ 分别对应 Fl-TP 分子内酯键及 DSPE-PEG 分子内酰胺键的 $\mathrm{C}=\mathrm{O}$ 的伸缩振动峰 ${ }^{[46,47]}$, 证明 Fl-TP-UCNPs 组装成功. TEM 图像表明在修饰前后, DSPE-PEG@UCNPs 与 Fl-TPUCNPs 的形貌均未发生明显变化(图 S4A, B). 由于
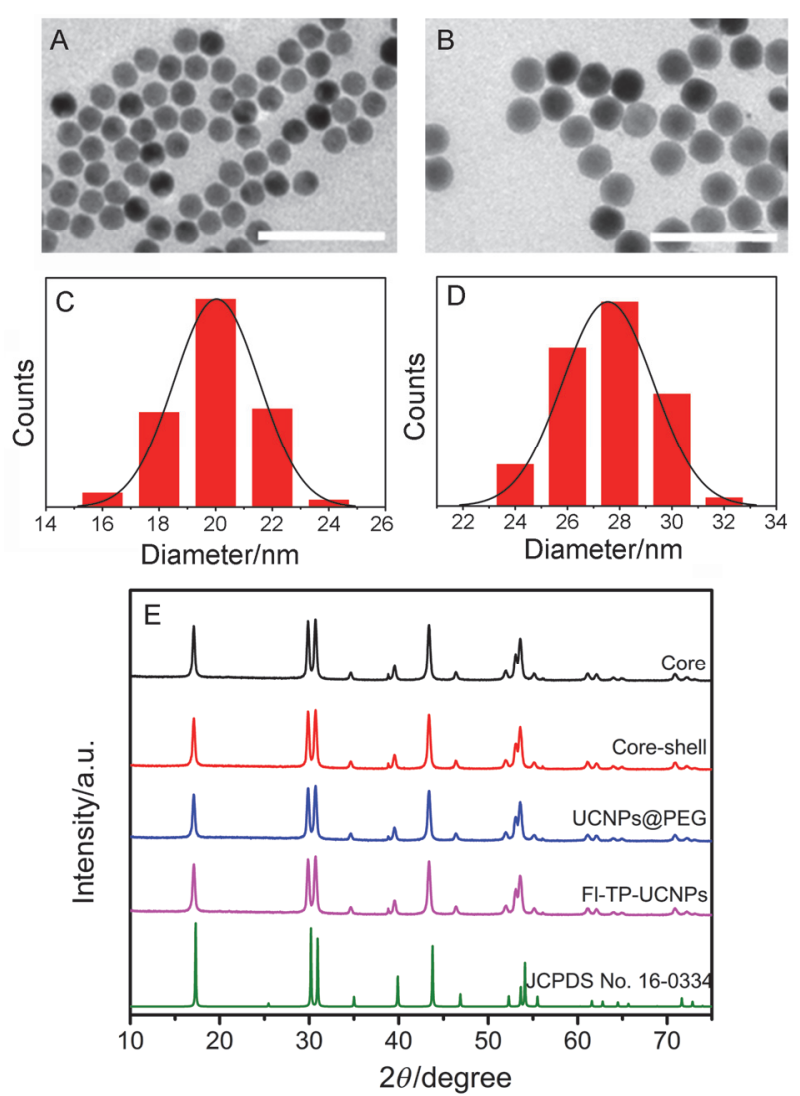

图 3 (A) $\mathrm{NaYbF}_{4}: \mathrm{Gd}$ 和(B) $\mathrm{NaYbF}_{4}: \mathrm{Gd} @ \mathrm{NaYF}_{4}: \mathrm{Yb}: \mathrm{Tm}$ 的透射电子显 微镜成像; (C) $\mathrm{NaYbF}_{4}: \mathrm{Gd}$ 和(D) $\mathrm{NaYbF}_{4}: \mathrm{Gd} @ \mathrm{NaYF}_{4}: \mathrm{Yb}: \mathrm{Tm}$ 的粒径分 布直方图; (E)核 $\mathrm{NaYbF}_{4}: \mathrm{Gd}$ 、核-壳 $\mathrm{NaYbF}_{4}: \mathrm{Gd} @ \mathrm{NaYF}_{4}: \mathrm{Yb}: \mathrm{Tm}$ 、 UCNPs@PEG 和 Fl-TP-UCNPs 的 XRD 图像

Figure 3 The transmission electron microcopy (TEM) images of OA-coated (A) $\mathrm{NaYbF}_{4}: \mathrm{Gd}$ and (B) $\mathrm{NaYbF}_{4}: \mathrm{Gd} @ \mathrm{NaYF}_{4}: \mathrm{Yb}: \mathrm{Tm}$. Histograms of the size distribution of (C) $\mathrm{NaYbF}_{4}: \mathrm{Gd}$ and (D) $\mathrm{NaYbF}_{4}: \mathrm{Gd} @$ $\mathrm{NaYF}_{4}: \mathrm{Yb}: \mathrm{Tm}$. (E) XRD patterns of the core structure $\mathrm{NaYbF}_{4}: \mathrm{Gd}$, core-shell structure $\mathrm{NaYbF}_{4}: \mathrm{Gd} @ \mathrm{NaYF}_{4}: \mathrm{Yb}: \mathrm{Tm}, \mathrm{UCNPs} @ \mathrm{PEG}$ and Fl-TP-UCNPs

Fl-TP 分子为电中性, 与 Fl-TP 结合之后, DSPE-PEG@UCNPs 的 Zeta 电势未发生显著变化(图 S5A, B). DSPE-PEG@UCNPs 的水合动力学直径为 150 $\mathrm{nm}$ (图 S5C), 与 Fl-TP 结合之后, 水合动力学直径变为 $190 \mathrm{~nm}$ (图 S5D). 在 $980 \mathrm{~nm}$ 连续激光激发下, 探针与 AS 反应后, $525 \mathrm{~nm}$ 处可以明显察到 Fl-HNO 的发射, UCNPs 在 $480 \mathrm{~nm}$ 处的发射强度相应地下降(图 S6), 由 此证明我们成功构建了一个基于 LRET 的比率型检测平 台.

\subsection{FI-TP-UCNPs 探针对 HNO 的响应}

随后我们探究了 Fl-TP-UCNPs 对 HNO 的响应性能. 首先, 对影响探针响应性能的因素进行优化, 图 S7 显示 了 Fl-TP-UCNPs 与 $\mathrm{HNO}$ 反应的动力学曲线. 由图可知 比率信号 $\left(F_{525 \mathrm{~nm}} / F_{480 \mathrm{~nm}}\right)$ 随时间逐渐增大, 在 $50 \mathrm{~min}$ 时 达到平台. 为确保反应完全, 将捊育时间定为 $1 \mathrm{~h}$. 染料 Fl-TP 的负载量对探针的响应性能也会产生显著影响, 如图 S8 所示, 染料浓度在 $60 \sim 300 \mu \mathrm{mol} \cdot \mathrm{L}^{-1}$ 范围时, 随 
染料浓度增加, 比率信号 $\left(F_{525} \mathrm{~nm} / F_{480 \mathrm{~nm}}\right)$ 显著增大. 当 Fl-TP 的浓度达到 $400 \mu \mathrm{mol} \cdot \mathrm{L}^{-1}$ 时, 此时 Fl-TP 的负载量 达到饱和, 比率信号增加趋势不明显. 因此, 选择 300 $\mu \mathrm{mol} \cdot \mathrm{L}^{-1}$ 作为构建探针的最优染料浓度.

在最佳条件下, 探究 Fl-TP-UCNPs 对 HNO 的响应. 如图 4A 所示, 在 $0 \sim 100 \mu \mathrm{mol} \cdot \mathrm{L}^{-1}$ 范围内, 随 $\mathrm{HNO}$ 浓 度增加, UCNPs 的苂光强度 $\left(F_{480 \mathrm{~nm}}\right)$ 逐渐降低, Fl-HNO 的苂光强度 $\left(F_{525 \mathrm{~nm}}\right)$ 逐渐增强. 比率信号 $\left(F_{525 \mathrm{~nm}} / F_{480 \mathrm{~nm}}\right)$ 在 $3 \sim 100 \mu \mathrm{mol} \cdot \mathrm{L}^{-1}$ 范围内与 $\mathrm{AS}$ 浓度对数呈良好的线 性关系 $\left(R^{2}=0.9914\right)$, 线性方程为 $F_{525} \mathrm{~nm} / F_{480 \mathrm{~nm}}=$ $-0.0493+0.25239 c$ ( $c$ 为 AS 浓度的对数) (图 4B), 检测 限 $\left(3 \sigma / s, s\right.$ 为线性方程斜率) 为 $23.4 \mathrm{nmol} \cdot \mathrm{L}^{-1}$, 与目前报 道检测 HNO 的有机小分子探针灵敏度相当(表 S1). 上述 结果表明该探针在水溶液中对 HNO 有较好的响应性能.
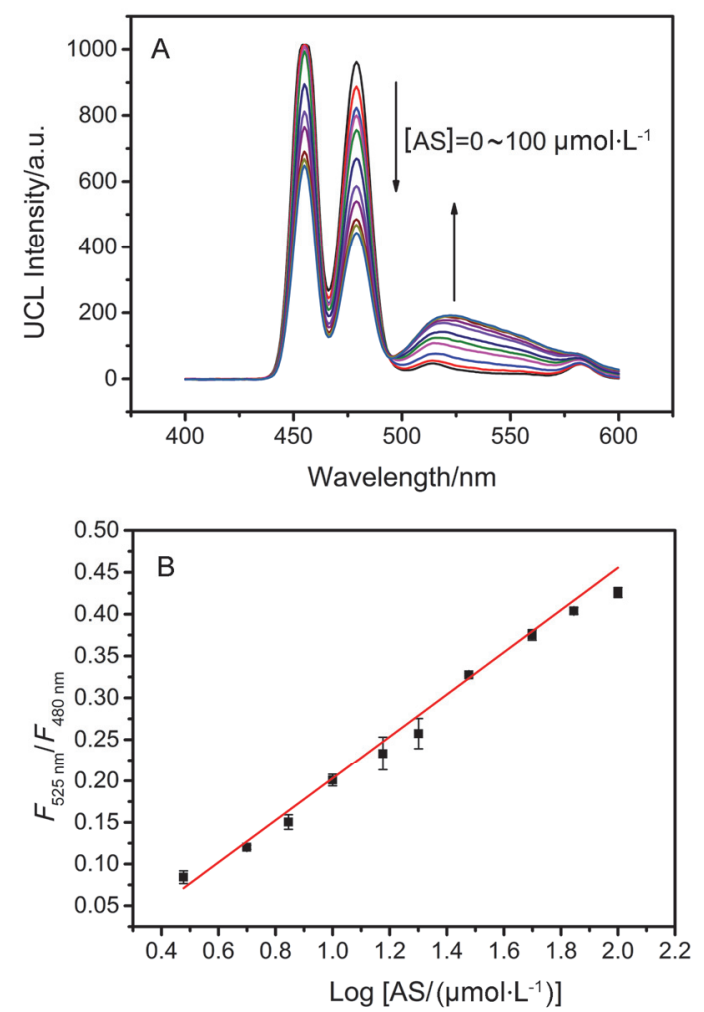

图 4 (A) Fl-TP-UCNPs 对不同浓度 AS 响应的发射光谱; (B) 比率信 号 $\left(F_{525 \mathrm{~nm}} / F_{480 \mathrm{~nm}}\right)$ 与 $\mathrm{AS}$ 浓度的对数之间的线性关系

Figure 4 (A) Emission spectra of Fl-TP-UCNPs responding to different concentrations of AS. (B) The linear relationship between ratiometric fluorescence signal $\left(F_{525 \mathrm{~nm}} / F_{480 \mathrm{~nm}}\right)$ of Fl-TP-UCNPs and the logarithm of AS concentration

\subsection{FI-TP-UCNPs 探针的选择性与稳定性}

为实现生物体系中的应用, 苂光探针需具备良好的 选择性与稳定性以克服复杂生物环境产生的干扰. 为考 察探针的选择性, 在相同条件下测试探针对其他干扰物 的响应. 如图 S9 所示, 金属离子、氨基酸、ROS、RSS(活 性硫类物质)、RNS 等干扰物对比率苂光信号的影响较
小, 探针对 $\mathrm{HNO}$ 有较好的选择性. 随后探究 Fl-TP-UCNPs 探针的热稳定性和 $\mathrm{pH}$ 稳定性. 如图 S10A 所示, 在 $37{ }^{\circ} \mathrm{C}$ 下孵育 $2 \mathrm{~h}$, 上转换荧光强度基本无变化. 此外, 如图 S10B 所示, 在 $4.5 \sim 8.0$ 的 $\mathrm{pH}$ 范围内, 探针 的上转换苂光强度基本保持稳定. 以上结果表明探针具 有良好的选择性、热稳定性和 $\mathrm{pH}$ 稳定性, 满足进行生 物体系中 HNO 成像分析的前提.

\section{6 细胞成像}

为确保探针具有足够的生物相容性, 首先采用 CCK-8 试验考察探针的细胞毒性. 如图 S11 所示, 在与 不同浓度 Fl-TP-UCNPs $\left(0 \sim 0.6 \mathrm{mg} \bullet \mathrm{mL}^{-1}\right)$ 孵育 $24 \mathrm{~h}$ 后, HeLa 细胞存活率无明显变化, 表明探针毒性较低, 适 合用于生物成像. 随后, 本工作考察了 Fl-TP-UCNPs 监 测活细胞中 HNO 含量变化的能力. Fl-TP-UCNPs $(0.3$ $\mathrm{mg} \cdot \mathrm{mL}^{-1}$ ) 探针与 $\mathrm{HeLa}$ 细胞孵育 $2 \mathrm{~h}$, 通过胞吞形式进入 细胞 ${ }^{[48]}$, 随后用不同浓度的 $\mathrm{AS}\left(0,10,50,100 \mu \mathrm{mol} \cdot \mathrm{L}^{-1}\right)$ 处理细胞 $1 \mathrm{~h}$ 后进行苂光成像. 如图 5A $\sim \mathrm{D}$ 所示, 随着 HNO 浓度增大, UCNPs 通道的苂光发射强度逐渐降低,

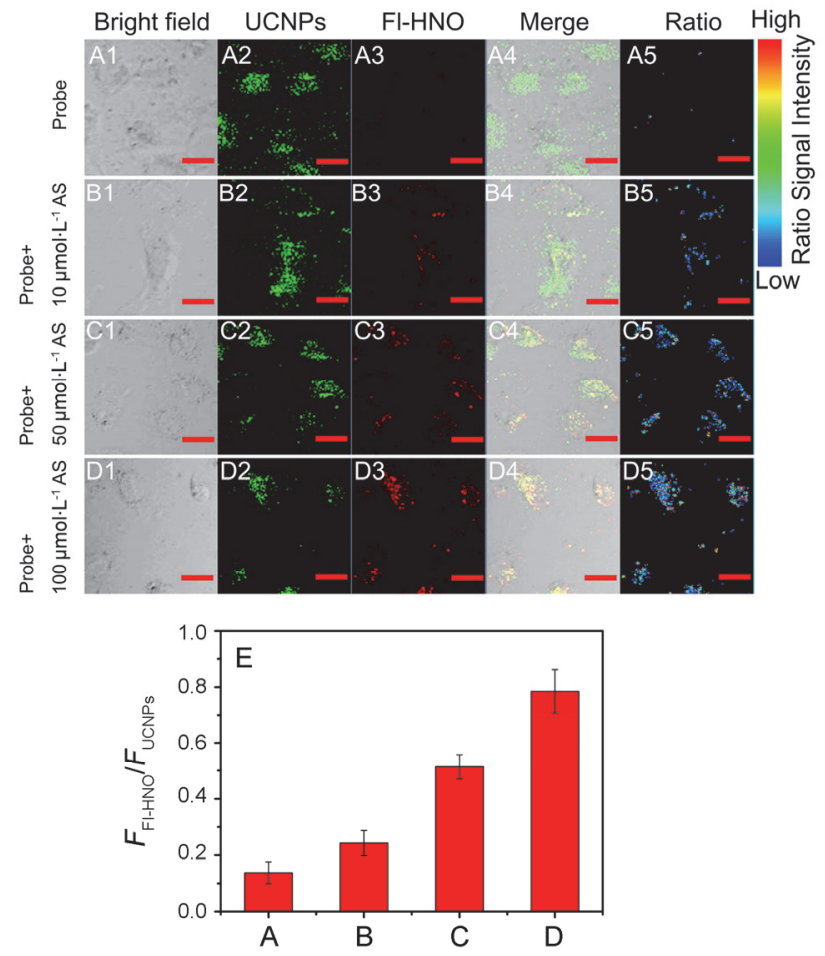

图 $5 \mathrm{HeLa}$ 细胞的共聚焦成像, $(\mathrm{A} \sim \mathrm{D})$ 用 Fl-TP-UCNPs $(0.3 \mathrm{mg}$ • $\mathrm{mL}^{-1}$ ) 孵育 $2 \mathrm{~h}$, 然后细胞用不同浓度的 $\mathrm{AS}\left(\mathrm{A}: 0 ; \mathrm{B}: 10 \mu \mathrm{mol} \cdot \mathrm{L}^{-1} ; \mathrm{C}\right.$ : $50 \mu \mathrm{mol} \cdot \mathrm{L}^{-1}$; D: $100 \mu \mathrm{mol} \cdot \mathrm{L}^{-1}$ )处理 $1 \mathrm{~h}$; (E) 图(A $\left.\sim \mathrm{D}\right)$ 的比率信号 $\left(F_{\mathrm{Fl}-\mathrm{HNO}} / F_{\mathrm{UCNPs}}\right)$. 收集 $400 \sim 500 \mathrm{~nm}$ (UCNPs 通道)和 $500 \sim 600 \mathrm{~nm}$ (Fl-HNO 通道)的信号, 比例尺: $20 \mu \mathrm{m}$

Figure 5 Confocal microscopic images of HeLa cells incubated with Fl-TP-UCNPs $\left(0.3 \mathrm{mg} \cdot \mathrm{mL}^{-1}\right)$ for $2 \mathrm{~h}$ : (A) cells incubated with Fl-TP-UCNPs only; $(B \sim D)$ cells treated with (B) $10 \mu \mathrm{mol} \cdot \mathrm{L}^{-1}$ AS; (C) $50 \mu \mathrm{mol} \cdot \mathrm{L}^{-1}$ AS; (D) $100 \mu \mathrm{mol} \cdot \mathrm{L}^{-1}$ AS after pretreated with Fl-TP-UCNPs. (E) Ratiometric signal of Fl-HNO emission to UCNPs emission in images $(A \sim D)$. The emissions were collected at $400 \sim 500$ $\mathrm{nm}$ (UCNPs channel) and 500 600 nm (Fl-HNO channel), respectively. Scale bar: $20 \mu \mathrm{m}$ 
Fl-HNO 通道的苂光发射强度逐渐增强, HNO 浓度为 $100 \mu \mathrm{mol} \cdot \mathrm{L}^{-1}$ 时, 比率信号达到最大(图 $5 \mathrm{E}$ ), 这与溶液 中的测试结果相符. 证明 Fl-TP-UCNPs 可应用于活细胞 内 $\mathrm{HNO}$ 浓度的变化监测.

根据文献报道, 机体内气体信号分子 $\mathrm{H}_{2} \mathrm{~S}$ 与 $\mathrm{NO}$ 相 互作用( “串扰” 机制)生成 $\mathrm{HNO}^{[49]}$. 本文初步探究了活 细胞中 $\mathrm{H}_{2} \mathrm{~S}$ 与 $\mathrm{NO}$ 相互作用生成 HNO 的过程. 文献报 道 $\mathrm{Na}_{2} \mathrm{~S}$ 和 DETA NONOate 可作为产生 $\mathrm{H}_{2} \mathrm{~S}$ 和 $\mathrm{NO}$ 的供 体 ${ }^{[50]}$, 因此使用二者探究该过程. 如图 6C 所示, 只有同 时添加了 $\mathrm{Na}_{2} \mathrm{~S}$ 与 DETA NONOate, 才可以观察到 UCNPs 通道信号减弱, Fl-HNO 通道信号增强. 而只使 用 Fl-TP-UCNPs 处理的图 6A、未使用 DETA NONOate 处理的图 $6 \mathrm{~B}$ 及使用 $N$-乙酰 - $L$ - 半胱氨酸 ( $N$-acetyl- $L$-cysteine, NAC, 颈基清除剂)处理的图 6D 中

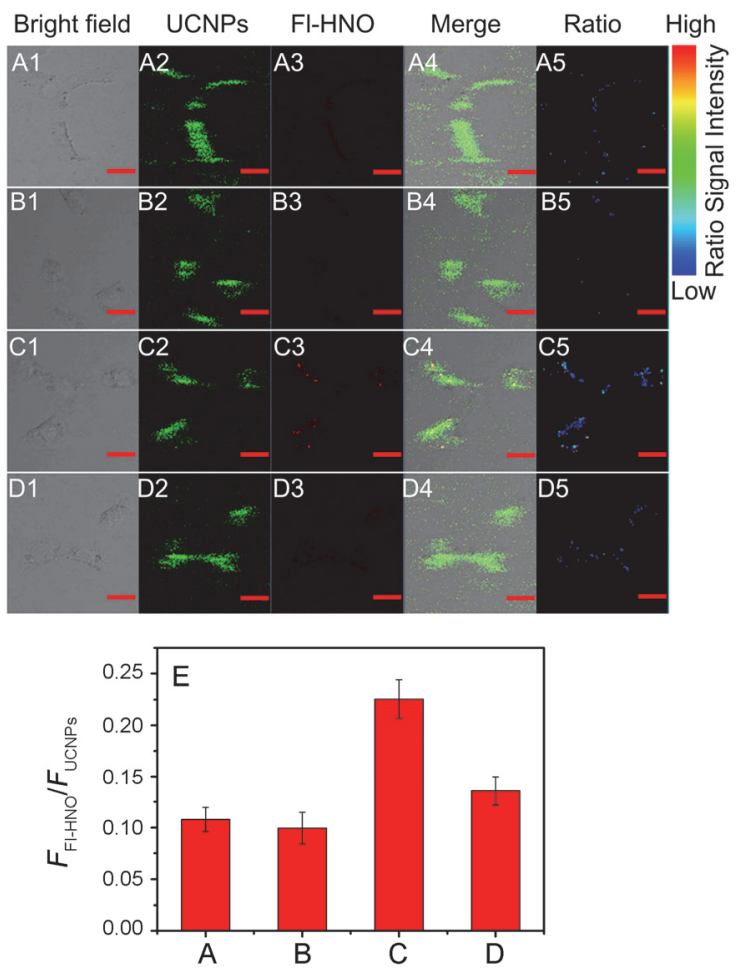

图 $6 \mathrm{HeLa}$ 细胞探针的共聚焦成像, $(\mathrm{A} \sim \mathrm{D})$ 用 Fl-TP-UCNPs $(0.3$ $\mathrm{mg} \cdot \mathrm{mL}^{-1}$ ) 捊育 $2 \mathrm{~h}$, 然后(A) 只用探针处理; (B) 用 $200 \mu \mathrm{mol} \cdot \mathrm{L}^{-1} \mathrm{Na}_{2} \mathrm{~S}$ 处理 $0.5 \mathrm{~h}$; (C) 用 $200 \mu \mathrm{mol} \cdot \mathrm{L}^{-1} \mathrm{Na}_{2} \mathrm{~S}$ 处理 $0.5 \mathrm{~h}$, 再用 $1 \mathrm{mmol} \cdot \mathrm{L}^{-1}$ DETA NONOate 处理 $1 \mathrm{~h}$; (D) $200 \mu \mathrm{mol} \cdot \mathrm{L}^{-1} \mathrm{Na}_{2} \mathrm{~S}$ 处理 $0.5 \mathrm{~h}$, 再加入 1 $\mathrm{mmol} \cdot \mathrm{L}^{-1} \mathrm{NAC}$ 处理 $0.5 \mathrm{~h}$, 最后再用 $1 \mathrm{mmol} \cdot \mathrm{L}^{-1}$ DETA NONOate 处理 $1 \mathrm{~h}$; (E) 图 $(\mathrm{A} \sim \mathrm{D})$ 的比率信号 $\left(F_{\mathrm{Fl}-\mathrm{HNO}} / F_{\mathrm{UCNPS}}\right)$. 收集 $400 \sim 500 \mathrm{~nm}$ (UCNPs 通道)和 500 600 nm (Fl-HNO 通道)的信号, 比例尺: $20 \mu \mathrm{m}$ Figure 6 Confocal microscopic images of HeLa cells incubated with Fl-TP-UCNPs $\left(0.3 \mathrm{mg} \cdot \mathrm{mL}^{-1}\right)$ for $2 \mathrm{~h}$ : (A) cells incubated with Fl-TP-UCNPs only; (B) cells pretreated with $200 \mu \mathrm{mol} \cdot \mathrm{L}^{-1} \mathrm{Na}_{2} \mathrm{~S}$ for 0.5 $\mathrm{h}$; (C) cells pretreated with $200 \mu \mathrm{mol} \cdot \mathrm{L}^{-1} \mathrm{Na}_{2} \mathrm{~S}$ for $0.5 \mathrm{~h}$ and $1 \mathrm{mmol} \cdot \mathrm{L}^{-1}$ DETA NONOate for another $1 \mathrm{~h}$; (D) cells pretreated with $200 \mu \mathrm{mol} \cdot \mathrm{L}^{-1}$ $\mathrm{Na}_{2} \mathrm{~S}$ for $0.5 \mathrm{~h}$ and then $1 \mathrm{mmol} \cdot \mathrm{L}^{-1} \mathrm{NAC}$ for another $0.5 \mathrm{~h}$, finally 1 $\mathrm{mmol} \cdot \mathrm{L}^{-1}$ DETA NONOate was added for $1 \mathrm{~h}$. (E) Ratiometric signal of Fl-HNO emission to UCNPs emission in images $(\mathrm{A} \sim \mathrm{D})$. Images were acquired at $400 \sim 500 \mathrm{~nm}$ (UCNPs channel) and 500 $600 \mathrm{~nm}$ (Fl-HNO channel), respectively. Scale bar: $20 \mu \mathrm{m}$
均只能观察到 UCNPs 通道出现苂光, Fl-HNO 通道荧光 信号不明显. 基于以上测试结果，证明 Fl-TP-UCNPs 可 用于活细胞中监测 $\mathrm{H}_{2} \mathrm{~S}$ 与 $\mathrm{NO}$ 共同作用产生的 $\mathrm{HNO}$, 有 望用于生理病理过程中 HNO 浓度的监测.

\section{7 组织成像}

为进一步考察 Fl-TP-UCNPs 在活体内的分析性能, 测试了 Fl-TP-UCNPs 对小鼠肝脏组织中 HNO 的响应能 力 $^{[51,52]}$. 因为根据文献报道, 粒径为 $20 \sim 150 \mathrm{~nm}$ 的纳米 颗粒在肝脏中富集较多, 所以选取肝脏进行成像 ${ }^{[53]}$. 将 Fl-TP-UCNPs $\left(200 \mu \mathrm{L}, 5 \mathrm{mg} \cdot \mathrm{mL}^{-1}\right.$ 分散于生理盐水)通过 腹腔注射至 Balb/c 小鼠(雄性, 约 $20 \mathrm{~g}$ ), $20 \mathrm{~min}$ 后腹腔注 射不同浓度的 $\mathrm{AS}$ 溶液(体积为 $100 \mu \mathrm{L}$, 物质的量分别为 $0,0.5 \mu \mathrm{mol}$ 和 $1 \mu \mathrm{mol}) .3 \mathrm{~h}$ 后将小鼠解剖并取肝脏组织, 切片后进行共聚焦成像. 如图 7 所示, 探针在肝脏组织 内均匀分布. 随着 AS 注射量的增加, UCNPs 通道的信 号逐渐减弱, Fl-HNO 通道的信号逐渐增强, 该现象与溶 液、细胞中的现象一致, 证明 Fl-TP-UCNPs 可以测定生 物组织中 HNO 的浓度变化.
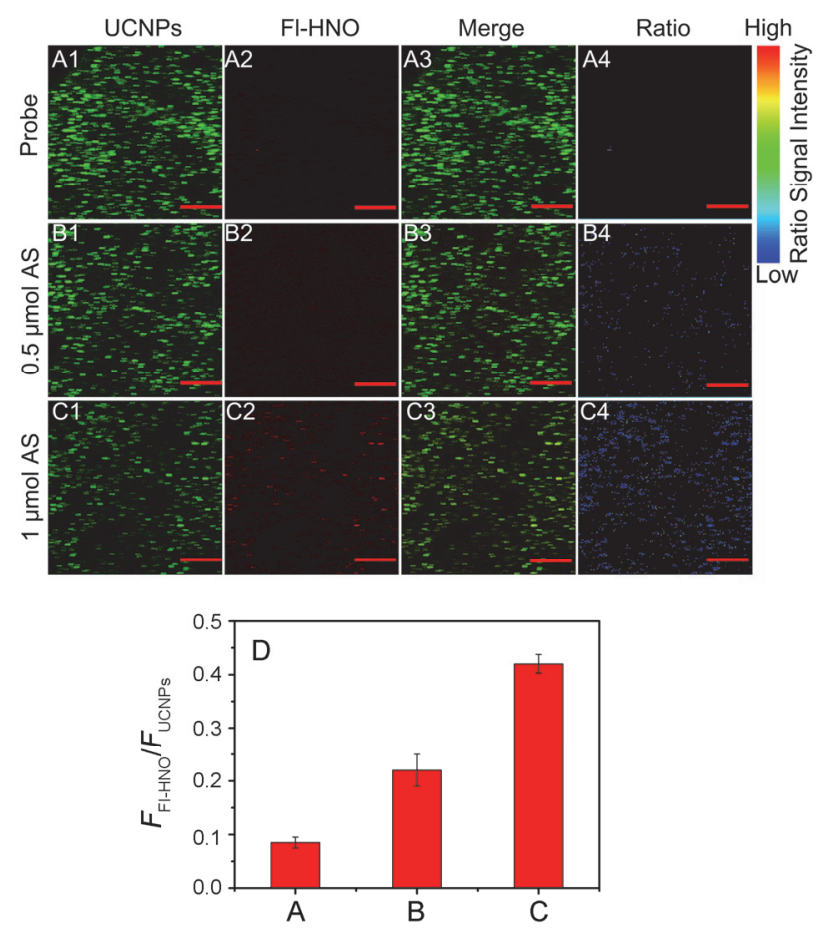

图 7 小鼠肝脏切片的共聚焦成像, $(\mathrm{A} \sim \mathrm{C})$ 用 Fl-TP-UCNPs $(0.3$ $\left.\mathrm{mg} \cdot \mathrm{mL}^{-1}\right)$ 探针对小鼠进行腹腔注射, 然后腹腔注射不同浓度的 AS (A: 0 ; B: $0.5 \mu \mathrm{mol} ; \mathrm{C}: 1 \mu \mathrm{mol})$; (D) 图 $(\mathrm{A} \sim \mathrm{C})$ 的比率信号 $\left(F_{\mathrm{Fl}-\mathrm{HNO}} / F_{\mathrm{UCNPs}}\right)$. 收集 $400 \sim 500 \mathrm{~nm}$ (UCNPs 通道)和 $500 \sim 600 \mathrm{~nm}$ (Fl-HNO 通道)的信号, 比例尺: $200 \mu \mathrm{m}$

Figure 7 Confocal microscopic images of mice liver slices injected with Fl-TP-UCNPs $\left(0.3 \mathrm{mg} \cdot \mathrm{mL}^{-1}\right)$. $(\mathrm{A} \sim \mathrm{C})$ Mice injected with AS $(\mathrm{A}: 0 \mu \mathrm{mol}$; B: $0.5 \mu \mathrm{mol}$; C: $1 \mu \mathrm{mol})$. (D) Ratiometric fluorescence signal of images $(A \sim C)$. The emissions were collected at $400 \sim 500 \mathrm{~nm}$ (UCNPs channel) and 500 600 nm (Fl-HNO channel), respectively. Scale bar: $200 \mu \mathrm{m}$. 


\section{3 结论}

成功构建了用于 $\mathrm{HNO}$ 检测的比率型上转换荧光纳 米探针. 该探针基于 LRET 原理, 以 UCNPs 为能量供体, 荧光素衍生物 Fl-TP 为潜在能量受体. 与 $\mathrm{HNO}$ 特异性反 应后, 由于触发了 LRET 作用, UCNPs 苂光强度减弱, 受体荧光逐渐增强, 比率信号 $\left(F_{525} \mathrm{~nm} / F_{480 \mathrm{~nm}}\right)$ 与 $\mathrm{AS}$ 浓度 对数间具有良好的线性关系. 该探针具有较高的灵敏度 及良好的稳定性、选择性、生物相容性, 成功实现了对 活细胞以及肝脏组织中 HNO 的检测和成像. 同时, 比 率信号也提高了检测结果的可靠性. 基于其优良性能, 该探针有望用于监测各种生理病理过程中 HNO 的含量 变化.

\section{4 实验部分}

\subsection{FI-TP 的合成}

合成路线如图 S12 所示. 化合物 $\mathbf{2}$ 和 $\mathbf{4}$ 按照文献报 道合成 ${ }^{[45,54]}$. 化合物 5 由化合物 $\mathbf{2}(200 \mathrm{mg}, 0.6 \mathrm{mmol})$ 和 化合物 $4(185 \mathrm{mg}, 0.72 \mathrm{mmol})$ 溶解于甲磺酸中, 在 $60{ }^{\circ} \mathrm{C}$ 反应 $8 \mathrm{~h}$, 再经过硅胶柱层析得到的. ${ }^{1} \mathrm{H}$ NMR (400 $\left.\mathrm{MHz}, \mathrm{CDCl}_{3}\right) \delta: 8.03(\mathrm{~d}, J=7.3 \mathrm{~Hz}, 1 \mathrm{H}), 7.71 \sim 7.58(\mathrm{~m}$, 2H), $7.16(\mathrm{~d}, J=7.5 \mathrm{~Hz}, 1 \mathrm{H}), 6.77(\mathrm{dd}, J=5.1,2.4 \mathrm{~Hz}$, $2 \mathrm{H}), 6.71 \sim 6.52(\mathrm{~m}, 4 \mathrm{H}), 3.98(\mathrm{t}, J=6.5 \mathrm{~Hz}, 2 \mathrm{H}), 1.84 \sim$ $1.73(\mathrm{~m}, 2 \mathrm{H}), 1.44(\mathrm{~d}, J=7.0 \mathrm{~Hz}, 2 \mathrm{H}), 1.26(\mathrm{~s}, 24 \mathrm{H}), 0.88$ (t, $J=6.8 \mathrm{~Hz}, 3 \mathrm{H}$ ).

FI-TP 通过下列合成步骤得到. 将 2-(二苯基膦)苯 甲酸 $(0.17 \mathrm{mmol})$ 溶于无水 $N, N$ 二 二甲基甲酰胺 $(N, N$-Dimethylformamide, DMF) 中. 在冰浴条件下, 加 入 $37 \mathrm{mg}(0.2 \mathrm{mmol}$ )的 1-乙基-(3-二甲基氨基丙基)碳酰 二亚胺盐酸盐 (1-Ethyl-3-(3-dimethylaminopropyl)carbodiimide hydrochloride, EDCI) 和 $2 \mathrm{mg}(0.02 \mathrm{mmol})$ 的 4-二甲氨基吡啶 (4-dimethylaminopyridine, DMAP). 反 应 $0.5 \mathrm{~h}$ 后, 加入 $110 \mathrm{mg}(0.2 \mathrm{mmol})$ 化合物 $\mathbf{5}$, 升温至室 温并摚拌过夜. 反应混合物倒入水中, 用乙酸乙酯萃取 后旋蒸除去有机溶剂, 最后用硅胶柱层析分离, 得到产 物 Fl-TP $64 \mathrm{mg}$, 产率为 $45 \% .{ }^{1} \mathrm{H}$ NMR $(400 \mathrm{MHz}$, $\left.\mathrm{CDCl}_{3}\right) \delta: 8.28 \sim 8.20(\mathrm{~m}, 1 \mathrm{H}), 8.01(\mathrm{~d}, J=7.4 \mathrm{~Hz}, 1 \mathrm{H})$, $7.58 \sim 7.69(\mathrm{~m}, 2 \mathrm{H}), 7.49 \sim 7.44(\mathrm{~m}, 2 \mathrm{H}), 7.38 \sim 7.26(\mathrm{~m}$, $10 \mathrm{H}), 7.14(\mathrm{~d}, J=7.5 \mathrm{~Hz}, 1 \mathrm{H}), 7.02 \sim 6.97(\mathrm{~m}, 1 \mathrm{H}), 6.95$ $(\mathrm{d}, J=2.2 \mathrm{~Hz}, 1 \mathrm{H}), 6.76 \sim 6.70(\mathrm{~m}, 2 \mathrm{H}), 6.69 \sim 6.64(\mathrm{~m}$, $1 \mathrm{H}), 6.64 \sim 6.57(\mathrm{~m}, 2 \mathrm{H}), 3.97(\mathrm{t}, J=6.5 \mathrm{~Hz}, 2 \mathrm{H}), 1.87 \sim$ $1.73(\mathrm{~m}, 2 \mathrm{H}), 1.51 \sim 1.40(\mathrm{~m}, 2 \mathrm{H}), 1.32 \sim 1.22(\mathrm{~m}, 24 \mathrm{H})$, $0.88(\mathrm{t}, J=6.8 \mathrm{~Hz}, 3 \mathrm{H})$ (图 S13). ${ }^{13} \mathrm{C}$ NMR $(101 \mathrm{MHz}$, $\left.\mathrm{CDCl}_{3}\right) \delta: 169.37,164.76,161.03,153.13,152.24,151.83$, $151.75,141.72,141.44,137.51,137.40,135.08,134.52$, 134.14, 134.13, 133.93, 133.92, 132.76, 131.43, 131.41, $129.79,128.93,128.87,128.86,128.66,128.64,128.58$, $128.57,128.38,126.51,125.05,124.08,117.45,116.73$,
$112.34,110.61,110.46,101.35,82.60,68.42,31.94,29.71$, 29.69, 29.67, 29.61, 29.58, 29.37, 29.09, 26.01, 22.71, 14.14 (图 S14). HRMS $(\mathrm{m} / \mathrm{z}):[\mathrm{M}+\mathrm{Na}]^{+}$calcd for $\left[\mathrm{C}_{55} \mathrm{H}_{57} \mathrm{O}_{6} \mathrm{NNa}\right]^{+}$867.3785, found 867.377 (图 S15).

\section{2 油酸修饰的上转换纳米颗粒(OA-UCNPs)合成}

UCNPs 根据逐层晶种生长法合成. 首先, 将 0.5 $\mathrm{mmol}$ 油酸盐 $\left[n\left(\mathrm{Yb}^{3+}\right): n\left(\mathrm{Gd}^{3+}\right)=70: 30\right], 20 \mathrm{mmol}$ 氟化 钠 $(\mathrm{NaF}), 10 \mathrm{~mL}$ 油酸(Oleic acid, $\mathrm{OA}$ )和 $10 \mathrm{~mL}$ 十八烯 (1-octadecene, ODE)加入到三口烧瓶中. 抽真空, 在氞 气保护下, 升温至 $110{ }^{\circ} \mathrm{C}$ 保持 $1 \mathrm{~h}$, 随后升温至 $320{ }^{\circ} \mathrm{C}$ 保持 $75 \mathrm{~min}$. 取出 $4 \mathrm{~mL}$ 反应液用于之后的表征, 然后 将分散在 $8 \mathrm{~mL} \mathrm{OA} / \mathrm{ODE}$ 混合液中的 $0.4 \mathrm{mmol}$ 油酸盐 $\left[n\left(\mathrm{Y}^{3+}\right): n\left(\mathrm{Yb}^{3+}\right): n\left(\mathrm{Tm}^{3+}\right)=75: 4: 1\right]$ 匀速加入到混 合液中, 继续保持 $320{ }^{\circ} \mathrm{C}$ 反应 $40 \mathrm{~min}$. 混合液冷却至室 温, 用等体积的乙醇使纳米颗粒沉淀下来, 纳米颗粒用 体积比为 $1: 1$ 的己烷/乙醇洗涤三次后, 分散在己烷里 备用.

\section{3 制备 FI-TP-UCNPs 探针}

将 $1 \mathrm{mg} \cdot \mathrm{mL}^{-1}$ 的 OA-UCNPs 和等质量的 DSPE-PEG 分散在氯仿中，溶液摚拌过夜直至氯仿挥发完全，将残 留物(DSPE-PEG@UCNPs)分散在超纯水中. Fl-TP 溶于 DMF 中, 然后缓慢滴加至搅拌中的 DSPE-PEG@ UCNPs 中, 过夜搅拌. 离心除去多余的 DSPE-PEG 和 Fl-TP, 沉淀用超纯水洗涤两次后并分散在超纯水中, 得 到 Fl-TP-UCNPs, 使其终浓度为 $1 \mathrm{mg} \cdot \mathrm{mL}^{-1}$.

\subsection{FI-TP-UCNPs 在水溶液中的响应}

在 HEPES $\left(100 \mathrm{mmol} \cdot \mathrm{L}^{-1}, \mathrm{pH}=7.2\right)$ 缓冲溶液中, $0.05 \mathrm{mg} \cdot \mathrm{mL}^{-1}$ 的 Fl-TP-UCNPs 与不同浓度的 AS $(0 \sim$ $100 \mu \mathrm{mol} \cdot \mathrm{L}^{-1}$ )混合, $37{ }^{\circ} \mathrm{C}$ 反应 $1 \mathrm{~h}$. 以 $980 \mathrm{~nm}$ 连续激光 器为激发光源, 收集 $400 \sim 600 \mathrm{~nm}$ 范围内的荧光信号.

\section{5 选择性}

在 HEPES $\left(100 \mathrm{mmol} \cdot \mathrm{L}^{-1}, \mathrm{pH}=7.2\right)$ 缓冲溶液中, $0.05 \mathrm{mg} \cdot \mathrm{mL}^{-1}$ 的 Fl-TP-UCNPs 与 AS $\left(100 \mu \mathrm{mol} \cdot \mathrm{L}^{-1}\right)$ 和 其他干扰物 $\left(100 \mu \mathrm{mol} \cdot \mathrm{L}^{-1}\right)$ 在 $37{ }^{\circ} \mathrm{C}$ 孵育 $1 \mathrm{~h}$. 以 $980 \mathrm{~nm}$ 连续激光器为激发光源, 收集 $400 \sim 600 \mathrm{~nm}$ 范围内的苂 光信号.

\section{6 稳定性}

在 HEPES $\left(100 \mathrm{mmol} \cdot \mathrm{L}^{-1}, \mathrm{pH}=7.2\right)$ 缓冲溶液中, $0.05 \mathrm{mg} \cdot \mathrm{mL}^{-1}$ 的 Fl-TP-UCNPs 在 $37{ }^{\circ} \mathrm{C}$ 孵育 $2 \mathrm{~h}$, 收集不 同时段的上转换荧光信号, 测定探针的热稳定性. 在磷 酸缓冲溶液内, 测定不同 $\mathrm{pH}$ 对探针的影响, $37{ }^{\circ} \mathrm{C}$ 条件 下稃育 $1 \mathrm{~h}$, 收集上转换苂光信号, 测定探针的 $\mathrm{pH}$ 稳定 性.

\section{7 细胞毒性}

探针的毒性用 CCK-8 的实验方法探究. 首先将 $\mathrm{HeLa}$ 细胞接种于 96 孔板中, 使用含有 $10 \%$ 胎牛血清和 
$1 \%$ 青霉素/链霉素的 DMEM 培养基, $37{ }^{\circ} \mathrm{C}$ 培养 $24 \mathrm{~h}$. 随 后加入含有不同浓度 Fl-TP-UCNPs 探针 $(0 、 0.1 、 0.2$ 、 $0.3 、 0.4 、 0.5 、 0.6 \mathrm{mg} \cdot \mathrm{mL}^{-1}$ )的新鲜培养基继续培养 $24 \mathrm{~h}$. 之后每孔加入 $10 \mu \mathrm{L}$ 的 CCK-8, 继续孵育 $4 \mathrm{~h}$. 最后用酶 标仪测定 $450 \mathrm{~nm}$ 处的吸光度, 计算细胞存活率, 细胞存 活率的计算方式为(Fl-TP-UCNPs 组的平均吸光度/对照 组的平均吸光度) $\times 100 \%$.

\section{8 细胞成像}

将 HeLa 细胞接种于共聚焦小皿中, 培养 $24 \mathrm{~h}$. 然 后用含有 $0.03 \mathrm{mg} \cdot \mathrm{mL}^{-1}$ 探针的新鲜培养基继续孵育 $2 \mathrm{~h}$. 用 PBS 清洗三次以除去未进入细胞的探针, 接着加入含 有不同浓度 $\mathrm{AS}\left(0 、 10 、 50 、 100 \mu \mathrm{mol} \cdot \mathrm{L}^{-1}\right)$ 的新鲜培养 基继续孵育 $1 \mathrm{~h}$. 用 PBS 清洗三次后，共聚焦成像.

接着探究该探针能否对气体分子 $\mathrm{H}_{2} \mathrm{~S}$ 与 $\mathrm{NO}$ 相互作 用产生的 HNO 成像. 将 HeLa 细胞接种于共聚焦小皿 中, 分为四组, 培养 $24 \mathrm{~h}$. 然后用含有 $0.03 \mathrm{mg} \bullet \mathrm{mL}^{-1}$ 探 针的新鲜培养基继续孵育 $2 \mathrm{~h}$. 第一组只使用 Fl-TP-UCNPs 处理 $2 \mathrm{~h}$. 第二组用 Fl-TP-UCNPs 处理后 继续用 $200 \mu \mathrm{mol} \cdot \mathrm{L}^{-1} \mathrm{Na}_{2} \mathrm{~S}$ 处理 $30 \mathrm{~min}$. 第三组重复第 二组的操作后再加入 $1 \mathrm{mmol} \cdot \mathrm{L}^{-1}$ DETA NONOate 孵育 $1 \mathrm{~h}$. 第四组依然重复第二组的操作, 在加入 DETA NONOate 前, 先用 $1 \mathrm{mmol} \bullet \mathrm{L}^{-1} \mathrm{NAC}$ 孵育 $30 \mathrm{~min}$. 最后, 用 PBS 清洗三次, 共聚焦成像.

\section{9 组织成像}

$\mathrm{Balb} / \mathrm{c}$ 小鼠(雄性, $20 \mathrm{~g}$ ) 由中国武汉古德生物科技有 限公司提供, 通过腹腔注射向小鼠 $(n=3)$ 体内注射 Fl-TP-UCNPs (200 $\mu \mathrm{L}, 5 \mathrm{mg} \cdot \mathrm{mL}^{-1}$ 分散于生理盐水). 20 $\min$ 后, 通过腹腔注射的方法向对应小鼠体内注射不同 浓度的 AS 溶液(体积为 $100 \mu \mathrm{L}$, 物质的量分别为 $0,0.5$ $\mu \mathrm{mol}$ 和 $1 \mu \mathrm{mol}) .3 \mathrm{~h}$ 后, 将小鼠解剖并取肝脏组织, 冰 切，共聚焦成像.

\section{References}

[1] Chen, X.; Tian, X.; Shin, I.; Yoon, J. Chem. Soc. Rev. 2011, 40, 4783.

[2] Chen, X.; Wang, F.; Hyun, J. Y.; Wei, T.; Qiang, J.; Ren, X.; Shin, I.; Yoon, J. Chem. Soc. Rev. 2016, 45, 2976.

[3] Fukuto, J. M; Switzer, C. H.; Miranda, K. M.; Wink, D. A. Annu. Rev. Pharmacol. Toxicol. 2005, 45,335 .

[4] Fukuto, J. M; Carrington, S. J. Antioxid. Redox. Signaling 2011, 14, 1649.

[5] Wong, P. S.-Y.; Hyun, J.; Fukuto, J. M.; Shirota, F. N.; Demaster, E. G.; Shoeman, D. W.; Nagasawa, H. T. Biochemistry 1998, 37, 5362.

[6] Jackson, M. I.; Fields, H. F.; Lujan, T. S.; Cantrell, M. M.; Lin, J.; Fukuto, J. M. Arch. Biochem. Biophys. 2013, 538, 120.

[7] Johnson, G. M.; Chozinski, T. J.; Gallagher, E. S.; Aspinwall, C. A.; Miranda, K. M. Free Radical Biol. Med. 2014, 76, 299.

[8] Nakagawa, H. Nitric Oxide. 2011, 25, 195.

[9] Fukuto, J. M.; Cisneros, C. J.; Kinkade, R. L. Inorg. Biochem. 2013, $118,201$.

[10] Switzer, C. H.; Flores-Santana, W.; Mancardi, D.; Donzelli, S.; Basudhar, D.; Ridnour, L. A.; Miranda, K. M.; Fukuto, J. M.; Paolocci, N.; Wink, D. A. Biochim. Biophys. Acta Bioenerg. 2009, 1787, 835.

[11] Shafirovich, V.; Lymar, S. V. Proc. Natl. Acad. Sci. U. S. A. 2002, 99,7340 .
[12] Li, X.; Gao, X.; Shi, W.; Ma, H. Chem. Rev. 2014, 114, 590.

[13] Yuan, L.; Lin, W.; Zheng, K.; Zhu, S. Acc. Chem. Res. 2013, 46 , 1462.

[14] Zhang, H.; Liu, R.; Tan, Y.; Xie, W. H.; Lei, H.; Cheung, H. Y.; Sun, H. ACS Appl. Mater. Interfaces 2015, 7, 5438.

[15] Rosenthal, J.; Lippard, S. J. J. Am. Chem. Soc. 2010, 132, 5536.

[16] Wrobel, A. T.; Johnstone, T. C.; Liang, A. D.; Lippard, S. J.; Rivera-Fuentes, P. J. Am. Chem. Soc. 2014, 136, 4697.

[17] Li, H.; Yao, Q.; Xu, F.; Xu, N.; Ma, X.; Fan, J.; Long, S.; Du, J.; Wang, J.; Peng, X. Anal. Chem. 2018, 90, 4641.

[18] Ali, F.; Sreedharan, S.; Ashoka, A. H.; Saeed, H. K.; Smythe, C. G. W.; Thomas, J. A.; Das, A. Anal. Chem. 2017, 89, 12087.

[19] Jiao, C.-P.; Liu, Y.-Y.; Lu, W.-J.; Zhang, P.-P.; Wang, Y.-F. Chin. J. Org. Chem. 2019, 39, 591 (in Chinese). (矫春鹏, 刘媛媛, 路文娟, 张平平, 王延风, 有机化学, 2019, 39, 591.)

[20] Wang, X.-F.; Wei, C.; Li, X.-Y.; Zheng, X.-Y.; Geng, X.-W.; Zhang, P.-Z.; Li, X.-L. Chin. J. Org. Chem. 2019, 39, 469. (in Chinese). (王 晓芬, 魏超, 李雪艳, 郑雪阳, 耿晓维, 张平竹, 李小六, 有机化 学, 2019, 39, 469.)

[21] Cline, M. R.; Toscano, J. P. J. Phys. Org. Chem. 2011, 24, 993.

[22] Zhou, Y.; Liu, K.; Li, J.-Y.; Fang, Y.; Zhao, T.-C.; Yao, C. Org. Lett. 2011, 13, 1290.

[23] Mcquade, L. E.; Lippard, S. J. Curr. Opin. Chem. Biol. 2010, 14, 43.

[24] Zheng, W.; Huang, P.; Tu, D.; Ma, E.; Zhu, H.; Chen, X. Chem. Soc. Rev. 2015, 44, 1379.

[25] Yang, D.; Ma, P.; Hou, Z.; Cheng, Z.; Li, C.; Lin, J. Chem. Soc. Rev. 2015, 44, 1416.

[26] Chen, G.; Qiu, H.; Prasad, P.; Chen, X. Chem. Rev. 2014, 114, 5161.

[27] Zhu, X.; Feng, W.; Chang, J.; Tan, Y.-W.; Li, J.; Chen, M.; Sun, Y.; Li, F. Nat. Commun. 2016, 7, 10437.

[28] Zhou, L.; Wang, R.; Yao, C.; Li, X.; Wang, C.; Zhang, X.; Xu, C.; Zeng, A.; Zhao, D.; Zhang, F. Nat. Commun. 2015, 6, 7350.

[29] Gu, B.; Zhang, Q. Adv. Sci. 2018, 5, 1700609.

[30] Su, Q.; Feng, W.; Yang, D.; Li, F. Acc. Chem. Res. 2017, 50, 32.

[31] Wang, S.; Liu, L.; Fan, Y.; El-Toni, A. M.; Alhoshan, M. S.; Li, D.; Zhang, F. Nano Lett. 2019, 19, 2418.

[32] Fan, Y.; Liu, L.; Zhang, F. Nano Today 2019, 25, 68

[33] Fan, Y.; Zhang, F. Adv. Optical. Mater. 2019, 7, 1801417.

[34] Li, X.; Guo, Z.; Zhao, T.; Lu, Y.; Zhou, L.; Zhao, D.; Zhang, F. Angew. Chem., Int. Ed. 2016, 55, 2464

[35] Liu, L.; Wang, S.; Zhao, B.; Pei, P.; Fan, Y.; Li, X.; Zhang, F. Angew. Chem., Int. Ed. 2018, 57, 7518.

[36] Lin, R.-Y.; Chen, Y.; Tao, G.-Y.; Pei, X.-J.; Liu, F.; Li, N. Acta Chim. Sinica 2017, 75, 1103 (in Chinese). (林若韵, 陈阳, 陶广宇, 裴晓 静, 刘锋, 李娜, 化学学报, 2017, 75, 1103.)

[37] Chen, K.; Han, B.-C.; Ji, S.-X.; Sun, J.; Gao, Z.-Z.; Hou, X.-F. Acta Chim. Sinica 2019, 77, 365 (in Chinese). (陈凯, 韩百川, 嵇思金金, 孙瑾，高振忠，侯贤锋，化学学报, 2019, 77, 365.)

[38] Ma, C.; Xu, X.; Wang, F.; Zhou, Z.; Liu, D.; Zhao, J.; Guan, M.; Lang, C. I.; Jin, D. Nano Lett. 2017, 17, 2858.

[39] Wen, S.; Zhou, J.; Zheng, K.; Bednarkiewicz, A.; Liu, X.; Jin, D. Nat. Commun. 2018, 9, 2415.

[40] Siefe, C.; Mehlenbacher, R. D.; Peng, C. S.; Zhang, Y.; Fischer, S.; Lay, A.; McLellan, C. A.; Alivisatos, A. P.; Chu, S.; Dionne, J. A. J. Am. Chem. Soc. 2019, 141, 16997.

[41] Wang, F.; Han, Y.; Lim, C. S.; Lu, Y.; Wang, J.; Xu, J.; Chen, H.; Zhang, C.; Hong, M.; Liu, X. Nature 2010, 463, 1061.

[42] Han, S.; Deng, R.; Xie, X.; Liu, X. Angew. Chem., Int. Ed. 2014, 53, 11702.

[43] Zhong, Y.; Tian, G.; Gu, Z.; Yang, Y.; Gu, L.; Zhao, Y.; Ma, Y.; Yao, J. Adv. Mater. 2014, 26, 2831.

[44] Min, Q.; Zhao, L.; Qi, Y.; Lei, J.; Chen, W.; Xu, X.; Zhou, D.; Qiu, J.; Yu, X. Nanoscale 2018, 10, 19031.

[45] Maeda, H.; Fukuyasu, Y.; Yoshida, S.; Fukuda, M.; Saeki, K.; Matsuno, H.; Yamauchi, Y.; Yoshida, K.; Hirata, K.; Miyamoto, K.; Angew. Chem., Int. Ed. 2004, 43, 2389.

[46] Cen, Y.; Wu, Y.-M.; Kong, X.-J.; Wu, S.; Yu, R.-Q.; Chu, X. Anal. Chem. 2014, 86, 7119.

[47] Tao, L.; Li, Z.; Wang, P.; Zhao, F.; Liu, Z. J. Am. Chem. Soc. 2018, 140,14696

[48] Zhao, F.; Zhao, Y.; Liu, Y.; Chang, X.; Chen, C.; Zhao, Y. Small 2011, 7, 1322.

[49] Yang, M.; Fan, J.; Sun, W.; Du, J.; Long, S.; Shao, K.; Peng, X. Chem. Commun. 2019, 55, 8583.

[50] Suarez, S. A.; Muñoz, M.; Alvarez, L.; Venâncio, M. F.; Rocha, W. R.; Bikiel, D. E.; Marti, M. A.; Doctorovich, F. J. Am. Chem. Soc. 
2017, 139, 14483

[51] Jing, X.; Yu, F.; Chen, L. Chem. Commun. 2014, 50, 14253.

[52] Li, J.-B.; Wang, Q.; Liu, H.-W.; Yin, X.; Hu, X.-X.; Yuan, L.; Zhang, X.-B. Chem. Commun. 2019, 55, 1758.
[53] Blanco, E.; Shen, H.; Ferrari, M. Nat. Biotechnol. 2015, 33, 941

[54] Liu, Y.; Chen, M.; Cao, T.; Sun, Y.; Li, C.; Liu, Q.; Yang, T.; Yao, L.; Feng, W.; Li, F. J. Am. Chem. Soc. 2013, 135, 9869.

(Cheng, B.) 